\title{
Qos Parameters of an Energy Efficient Multicast Congestion Control Protocol (Eemcep) Over Fading Channels
}

\author{
Dr. M .Rajanbabu ${ }^{1}$, Ms .A.R .Sushma ${ }^{2}$, Ms. B.S.Sowjanya ${ }^{3}$, Mr. B .Suresh \\ kumar $^{4}$, Ms. B. Tejaswini ${ }^{5}$ \\ ${ }_{1,2,3,4,5}$ (Electronics \& Communication, Lendi Institute Of Engineering And Technology, India)
}

\begin{abstract}
In this paper we propose an energy efficient multicasting congestion control protocol for mobile ad hoc networks (MANETs) over fading channels. Our proposed scheme overcomes the disadvantages of existing multicast congestion control protocols which depend on individual receivers to detect congestion and adjust their receiving rates over fading channels. In the first phase we observe the performance of data transmission over wireless channels is well captured by their bit error rate which is a function of the signal to noise ratio at the receiver. These models are a function of the distance between the sender and the receiver, the path loss exponent, and the channel gain. The channel gain which is a time-variation parameter is modeled by probability distribution functions. So we mainly described the three most important and commonly used distribution functions for this probability distribution, i.e., AWGN, Rayleigh, and Racian models. In the second phase we proposed a protocol on these noise channels, the protocol requires to build a multicast tree routed at the source, by including the nodes with higher residual energy towards the receivers. In the third phase, we propose an admission control scheme in which a multicast flow is admitted or rejected depending upon on the output queue size. In the fourth phase, we propose a scheme which adjusts the multicast traffic rate at each bottleneck of a multicast tree. Because of the on-the-spot information collection and rate control, this scheme has very limited control traffic overhead and delay. Moreover, the proposed scheme does not impose any significant changes on the queuing, scheduling or forwarding policies of existing networks. Simulation results shows that our proposed protocol has better delivery ratio and throughput with less delay and energy consumption when compared with existing protocols.
\end{abstract}

\section{Introduction}

The bandwidth of WLANs is limited because they rely on an inexpensive, but error prone physical medium i.e., air. Hence it is important to evaluate the performance of wireless devices by considering the transmission characteristics, wireless channel parameters, and device structure. The performance of data transmission over wireless channels is well captured by observing their bit error rate which is a function of the signal to noise ratio at the receiver. Several models have been already proposed and investigated to calculate the signal to noise ratio in wireless channels. The channel gain which is a time-variation parameter is modeled by probability distribution functions.

A mobile ad-hoc network (MANET) is composed of mobile nodes without any infrastructure. Mobile nodes self-organize to form a network over radio links. These include military battlefields, emergency search, rescue sites, classrooms and conventions, where participants share information dynamically using their mobile devices. Multicasting is aimed to deliver data to a set of selected receivers. There is no restriction on the location or number of members in a host group. The important member identifications and functions are: group member, sources, destination, forwarding nodes, non-group member. The group membership is dynamic means that hosts may join and leave groups at any time Multicast packets are delivered to each member of a multicast group with the same best-efforts reliability and performance as unicast packets to members. Multicast groups may be of arbitrary size, may change membership dynamically, and may have either a global or local scope. The senders do not need to know membership groups, and needs not to be a member of that group. [2]. In addition, within a wireless medium, it is crucial to reduce the transmission overhead and power consumption. Multicasting can improve the efficiency of the wireless link when sending multiple copies of messages by exploiting the inherent broadcast property of wireless transmission. Hence, energy efficient routing plays a significant role in MANETs [1].Unlike typical wired multicast routing protocols, multicast routing for MANETs must address a diverse range of issues due to the characteristics of MANETs, such as low bandwidth, mobility and low power. MANETs deliver lower bandwidth than wired networks; therefore, the information collection during the formation of a routing table is expensive [1]. 


\section{Design of EEMCCP Fading Channel}

\section{Discussion}

\subsection{IEEE 802.11b Physical Layer Transmission Model}

Fig 2.1 shows a block diagram of the IEEE 802.11b PHY layer signal processing. All 802.11 standard senders use a scrambler before sending the data. This data whitener (scrambler and descrambler) uses a length-127 frame-synchronous scrambler followed by a $32 / 33$ bias-suppression encoding to randomize the data and to minimize the data DC bias and maximum run lengths. The frame synchronous scrambler uses the generator polynomial $S(x)$ as given in $S(x)=x^{7}+x^{4}+1$ and is illustrated in Fig 2.1

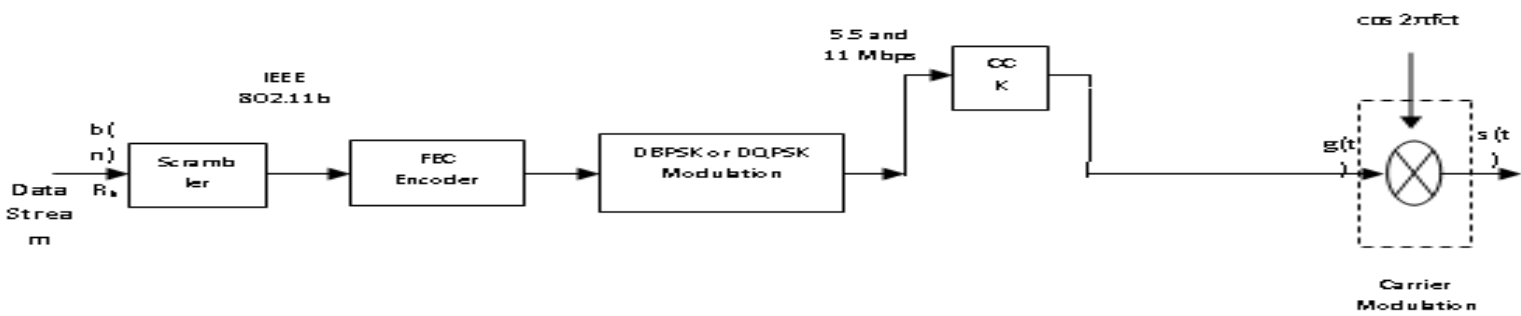

Fig2.1 The structure of receiver for 1 and $2 \mathrm{Mbps}$ has the de-spreading building block which uses the same Barker codes. This structure depends on coherent or differentially detection of signal as well.

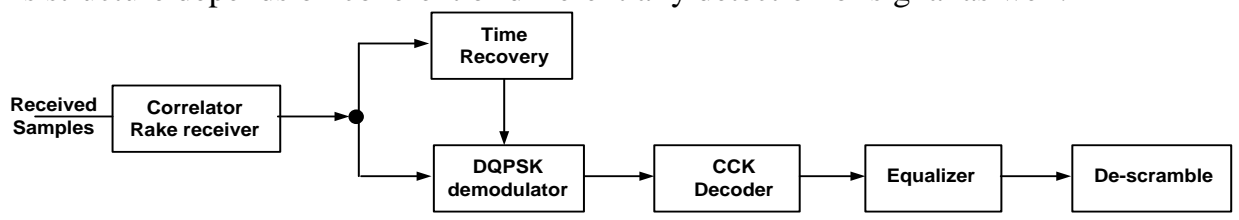

Figure 2.2 Block diagram of the IEEE $802.11 \mathrm{~b}$ receiver PHY layer

\subsection{Additive White Gaussian Noise (AWGN) Channel}

An AWGN channel adds white Gaussian noise to the signal that passes through it. It is the basic modelfor a digital communication channel and therefore used as a standard channel model. In this model, the transmitted signal gets disturbed by only a simple additive white Gaussian noise process. Thus the received signal can be obtained by:

$$
y(t)=s(t)+n(t)
$$

Where $n(t)$ denotes a sample function of the additive white Gaussian noise process with power spectral density of $\Phi_{n n}(f)=\frac{1}{2} N_{o} \mathrm{~W} / \mathrm{Hz}$ (John G. Proakis., 1995). The white noise is a noise which has a frequency spectrum that is continuous and uniform over a specified frequency band. In AWGN channel model, there is no random scattering and diffraction transmitted signals at the receiver. Unfortunately, most wireless channel links cannot be modeled as AWGN channels. In the next section the wireless fading channel which is more realistic is explained.

\subsection{Fading Channel}

Multipath fading is due to the constructive and destructive combination of randomly delayed, reflected, scattered and diffracted signal components. This type of fading is relatively fast and is therefore responsible for the short-term signal variations (M. K. Simon and M. S. Alouini., 2004). Depending on the nature of the radio propagation environment, there are different models describing the statistical of the multipath fading channel like Rayleigh, Nakagami-q, Nakagami-n (Rice) and Nakagami-m model. The Rayleigh distribution is frequently used to model multipath fading with no direct line of sight (LOS) path. In this case, the channel fading amplitude $\alpha$ is distributed according to

$$
P_{\alpha}(\alpha)=\frac{2 \alpha}{\Omega} \exp \left(-\frac{\alpha^{2}}{\Omega}\right)
$$

Where $\alpha$ is a random variable with mean-square value $\Omega=\bar{\alpha}^{2}$. In this model, the instantaneous SNR per symbol of the channel $\gamma$ is distributed according to an exponential distribution given by 


$$
P_{\gamma}(\gamma)=\frac{1}{\bar{\gamma}} \exp \left(-\frac{\gamma}{\bar{\gamma}}\right) \quad \gamma \geq 0
$$

Similarly, the Rice distribution which is often used to model propagation paths consisting of one strong direct LOS component can be characterized by:

$$
P_{\alpha}(\alpha)=\frac{2\left(1+n^{2}\right) e^{-n^{2}} \alpha}{\Omega} \exp \left(-\frac{\left(1+n^{2}\right) \alpha^{2}}{\Omega}\right) I_{0}\left(2 n \alpha \sqrt{\frac{1+n^{2}}{\Omega}}\right),{ }_{\alpha \geq 0}
$$

Where $n_{\text {is the Nakagami- }} n$ fading parameter, which ranges from 0 to $\infty$. $I_{0}($.$) is the zero order modified Bessel$ function of the first kind. The SNR of the channel, $\gamma$, is distributed (i.e. $P_{\gamma}$ ) according to a non central chisquare distribution given by,

$$
P_{\gamma}(\gamma)=\frac{\left(1+n^{2}\right) e^{-n^{2}}}{\bar{\gamma}} \exp \left(-\frac{\left(1+n^{2}\right) \gamma}{\bar{\gamma}}\right) I_{0}\left(2 n \sqrt{\frac{\left(1+n^{2}\right) \gamma}{\bar{\gamma}}}\right), \gamma \geq 0
$$

Similar distributions can be found in (M. K. Simon and M. S. Alouini., 2004) for other fading models. In this analysis, the Rayleigh fading model which is the most frequently used model for multipath wireless channels is used.

Generally, the performance of communication systems over such wireless channels is captured and analyzed using the probability of Bit Error Rate (BER) as a function of Signal to Noise and Interference Ratio (SINR). The BER decreases for high SNR at the receiver. However, the actual performance of the wireless system depends on several implementation issues and wireless channel characteristics.

\subsection{IEEE 802.11b Performance Evaluation in data transmission}

Four transmission modes are defined in 802.11b. In this section, the performance of these transmission modes is evaluated.

\section{1and 2 Mbps Transmission Modes}

In this section, the BER is calculated for 1 and $2 \mathrm{Mbps}$ transmission modes used in IEEE 802.11b WLANs. As explained in Section 2.8, for 1 and 2 Mbps transmission modes the modulations could be DBPSK and DQPSK or DE-BPSK and DE-QPSK according to receiver structure. It can be shown that in AWGN with Gray mapping, the probability of bit error for DE-BPSK and DE-QPSK are (M. K. Simon., et.al., 1995 and John G. Proakis., 1995):

$$
\begin{aligned}
P_{b}{ }^{D E-B P S K} & =P_{b}^{D E-Q P S K} \\
= & 2 Q\left(\sqrt{2 \frac{\varepsilon_{b}}{N_{o}}}\right)-2 Q^{2}\left(\sqrt{2 \frac{\varepsilon_{b}}{N_{o}}}\right)
\end{aligned}
$$

Similar to the calculations performed in Section 4.4.2 and 4.4.3, if the fading process is assumed to be Rayleigh distributed with $L$ resolvable i.i.d. paths, it can be shown that with maximum-ratio diversity combining, the fading-averaged BER for DE-BSPK and DE-QPSK can be obtained by (M. K. Simon and M. S. Alouini., 2004).

$$
\begin{aligned}
& P_{b}=2\left[\frac{1-\mu_{c}}{2}\right]^{L} \sum_{l=0}^{L-1}\left(\begin{array}{c}
L-1+l \\
l
\end{array}\right)\left[\frac{1-\mu_{c}}{2}\right]^{l}-2 X\left\{\frac{1}{4}-\frac{\mu_{c}}{\pi}\left[\left(\frac{\pi}{2}-\tan ^{-1} \mu_{c}\right) \sum_{l=0}^{L-1} \frac{\left(\begin{array}{c}
2 l \\
l
\end{array}\right)}{\left[4\left(1+\bar{\gamma}_{b}\right)\right]^{l}}\right.\right. \\
& \left.\left.-\sin \left(\tan ^{-1} \mu_{c}\right) \sum_{l=1}^{L-1} \sum_{i=1}^{l} \frac{T_{i l}}{\left(1+\bar{\gamma}_{b}\right]^{l}}\left[\cos \left(\tan ^{-1} \mu_{c}\right)\right]\right]^{2(l-i)+1}\right\} \\
& \text { Where } T_{i l}=\frac{\left(\begin{array}{c}
2 l \\
l
\end{array}\right)}{\left(\begin{array}{c}
2(l-i) \\
l-i
\end{array}\right)\left\{4^{i}[2(l-i)+1]\right\}} \text { and } \mu_{c}=\sqrt{\frac{\bar{\gamma}_{b}}{1+\bar{\gamma}_{b}}} \text {. }
\end{aligned}
$$

For the non-coherent detection receiver, i.e. DBPSK and DQPSK, the probability of bit error for AWGN channel is: 


$$
P_{b}^{D B P S K}=\frac{1}{2} \exp \left(-\frac{\gamma_{b}}{2}\right)
$$

and

$$
P_{b}^{D Q P S K}=\frac{1}{2}\left(1-Q_{1}(b, a)+Q_{1}(a, b)\right)
$$

Where $a=\sqrt{2 \gamma_{b}(1-\sqrt{2} / 2)}$ and $b=\sqrt{2 \gamma_{b}(1+\sqrt{2} / 2)}$. For the receiver with maximum ratio diversity combining the probability of bit error for DBPSK and DQPSK can be obtained by (John G. Proakis., 1995):

$$
P_{b}=\frac{1}{2}\left[1-\frac{\mu_{c}}{\sqrt{2-\mu_{c}^{2}}} \sum_{l=0}^{L-1}\left(\begin{array}{l}
2 l \\
l
\end{array}\right)\left(\frac{1-\mu_{c}^{2}}{4-2 \mu_{c}^{2}}\right)^{l}\right],
$$

Where $\mu_{c}=\frac{\bar{\gamma}_{b}}{1+\bar{\gamma}_{b}}$ for DBPSK and $\mu_{c}=\frac{2 \bar{\gamma}_{b}}{1+2 \bar{\gamma}_{b}}$ for DQPSK.

\subsection{5 and 11 Mbps Transmission Modes}

For the high data rate transmission modes in IEEE $802.11 \mathrm{~b}$, i.e. 5.5 and $11 \mathrm{Mbps}$, the CCK and binary convolution coding have been adopted as explained in section. The latter one is an optional transmission mode in $802.11 \mathrm{~b}$. In this section, the performance of 5.5 and 11 Mbps transmission modes with the CCK modulation is evaluated. The performance of CCK modulation (i.e., BER) directly depends on the equalizer . Basically there are three common equalizers: linear equalizer (LE), decision feedback equalizer (DFE), and maximum likelihood sequence estimator (MLSE). Note that it is too complex to calculate the closed form equations for BER calculations for CCK modulation. In this dissertation the performance evaluation of CCK modulation is evaluated for the MLSE equalizer. The simulation results for MLSE and DFE detections are compared (C. Jonietz., et.al., 2004). To evaluate the performance of CCK modulation in $802.11 \mathrm{~b}$, first calculate the pair wise error probability (PEP). PEP or $P_{e}(\alpha, \beta)$ is the probability that a code word $c^{\alpha}$ is transmitted and that the maximum likelihood receiver decides in favor of $c^{\beta}$ where $\alpha \neq \beta$. The authors (R. Schober., et.al. 20030) shows that $P_{e}(\alpha, \beta)$ can be calculated by

$$
P_{e}(\alpha, \beta)=\int_{x=-\infty}^{0} P_{\Delta(\alpha, \beta)}(x) d x
$$

Where $\Delta(\alpha, \beta)$ is a random variable which depends on the mean and the autocorrelation matrix of $h_{\text {(i.e., }}$ the channel impulse response (CIR) between transmit and receive antenna) with pdf $P_{\Delta(\alpha, \beta)}$. Then the union bound on the average bit error rate can be calculated by

$$
P_{b} \leq \frac{1}{|C|} \sum_{\substack{\alpha=1 \\ \beta=1 \\ \beta \neq \alpha}}^{|C|} \frac{n(\alpha, \beta)}{8} X P_{e}(\alpha, \beta)
$$

Where $n(\alpha, \beta)$ denotes the number of bit errors if $c^{\alpha}$ is transmitted and $c^{\beta}$ is detected.

The performance evaluation of the IEEE 802.11b transmission modes over AWGN and Rayleigh fading channels is done by calculating the probability of bit error. The receiver structure for IEEE $802.11 \mathrm{~b}$ mobile stations has been discussed and evaluated the performance of the transmission modes for multilink receiver stations. The performance of the CCK modulation in IEEE 802.11b is also discussed. The simulation results show that the performances of various transmission modes are highly dependent on the wireless channel model, the type of signal detection (coherent or differential detection), the type of encoders and decoders, the number of receiving antennas, modulation techniques and data rates. In this thesis, different modulation techniques performance with various data rates (1 Mbps and $2 \mathrm{Mbps}$ ) is observed for the designed fading channels. It is concluded that BPSK modulation in AWGN channel gives the lower probability of bit error (0.01222). But for real time scenarios fading channels are most common. In IEEE 802.11b standard, BPSK modulation with Rayleigh fading channel $(\mathrm{L}=2)$ at $1 \mathrm{Mbps}$ data rate gives the bit error probability of 0.02222 . These 
observations are useful in the design of the EEMCC protocol which is implemented under Rayleigh fading channel.
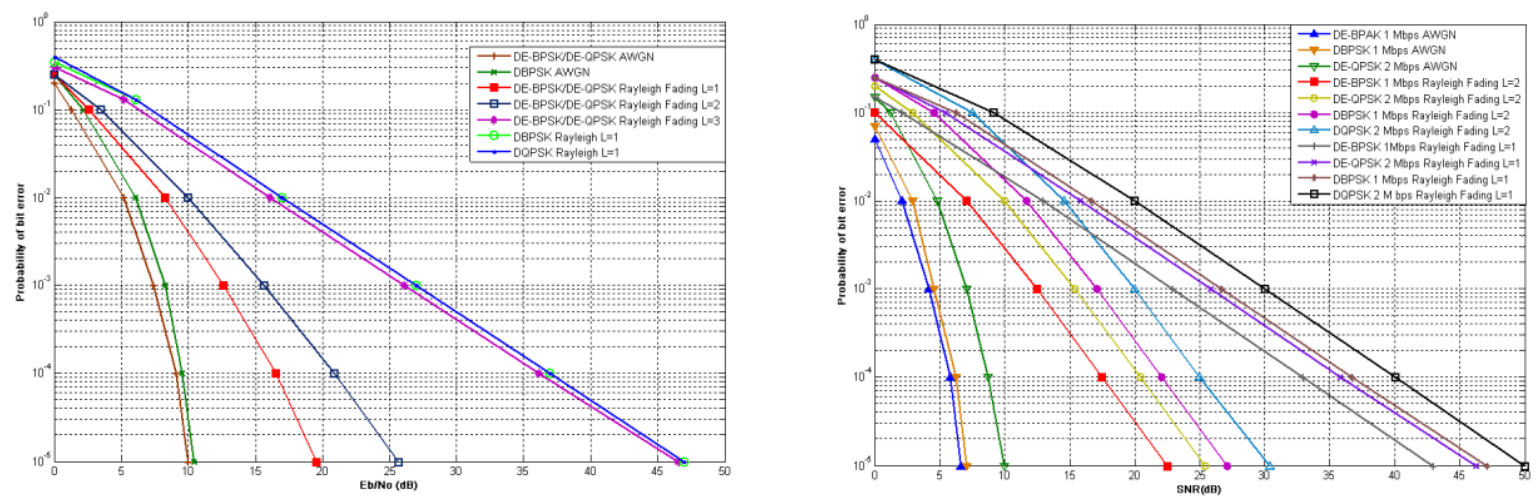

Figure 1:Probability of bit error for DE-BPSK, DE-QPSK, DBPSK, and DQPSK modulation techniques.

Figure2: Performance evaluation of 1 and 2 Mbps transmission modes in IEEE 802.11b using various modulation techniques .

\section{Design of Energy Efficient Multicast Congestion Control Protocol (EEMCCP) \\ Energy Efficient Tree Construction}

In our energy efficient multicast congestion control protocol we build a multicast tree routed at the source towards the receivers. The distance i.e. the geographical location of the nodes is assumed. Their residual energy is measured. The nodes are sorted based on its location from the source and arranged in a sequence order. A threshold value $\mathrm{P}$ is set and the nodes which are less than $\mathrm{P}(\mathrm{m}<\mathrm{P})$ are unicast from the source and the nodes which are greater $\mathrm{P}(\mathrm{m}>\mathrm{P})$ are multicast. In case of multicasting the node which has the minimum energy per corresponding receiver is set as the relay node. The relay node then forwards the packets from the source to the corresponding receivers.

Calculating Residual Energy of a Node : Consider a network with multicast groups S1, S2

Sx. Each group $\{\mathrm{Si}\}$ consists of $\mathrm{N}$ nodes. Every node in the MANET calculates its remaining energy periodically. The nodes may operate in either transmission or reception mode. Let $\left\{\mathrm{e}_{1}, \mathrm{e}_{2} \ldots \ldots \ldots . \mathrm{e}_{\mathrm{n}}\right\}$ are the residual energies of the nodes measured by the following method.

The power consumed for transmitting a packet is given by the Eq (2.12)

Consumed energy $=\mathrm{TP}^{*} \mathrm{t}$

Where TP is the transmitting power and $\mathrm{t}$ is transmission time.

The power consumed for receiving a packet is given by $\mathrm{Eq}(2.13)$

Consumed energy $=\mathrm{RP} * \mathrm{t}$

Where $\mathrm{RP}$ is the reception power and $\mathrm{t}$ is the reception time.

The value $\mathrm{t}$ can be calculated as

$\mathrm{t}=\mathrm{Ds} / \mathrm{Dr}$

Ds is Data size and Dr is Data rate

Hence, the residual energy (E) of each node can be calculated using Eq (2.12) or Eq (2.13) and Eq (2.14)

$E=$ Current energy - Consumed energy

Algorithm

1. Consider a group $\mathrm{Sj}=\left\{\mathrm{n}_{1}, \mathrm{n}_{2} \ldots \ldots \ldots . \mathrm{n}_{\mathrm{n}}\right\}$

2. Measure the distance $d$ of each node from source $A$ $\mathrm{d}(\mathrm{A}, \mathrm{ni})$ where $\mathrm{i}=1,2 \ldots \ldots \ldots . \mathrm{n}$

3. Sort the nodes $n_{i}$ in ascending order of $d$.

4. Create the partitions $\mathrm{X} 1$ and $\mathrm{X} 2$ of the nodes Ni such that

$$
\begin{aligned}
& \mathrm{X} 1=\left\{\mathrm{n}_{1} \ldots \ldots \ldots \mathrm{n}_{\mathrm{Q}}\right\} \\
& \mathrm{X} 2=\left\{\mathrm{n}_{\mathrm{Q}+1} \ldots \ldots \ldots . \mathrm{n}_{\mathrm{n}}\right\}
\end{aligned}
$$

Where $\mathrm{Q}$ is the distance threshold.

5. Source unicast the packets to X1

6. In X2 find a relay node $\mathrm{n}_{\mathrm{r}}$ which has $\max \left(\mathrm{E}_{\mathrm{i}}\right)$

7. Then A unicast the packets to $n_{r}$ which in turn multicast the packets to the rest of the nodes in X2. 


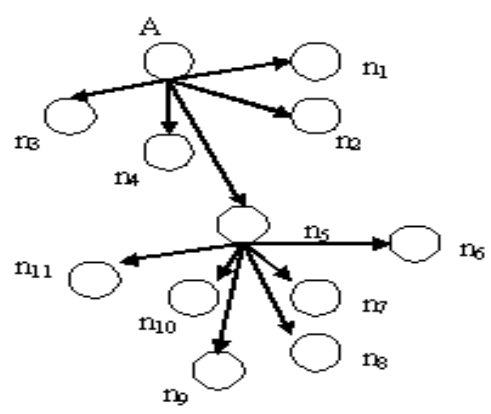

Figure 3.1: Energy efficient tree construction

Source A unicast the packets to nodes $n 1, n 2, n 3, n 4, n 5$ is the relay node. $n 5$ multicast the packets to the rest of the nodes $\mathrm{n}_{6} \ldots \ldots . . \mathrm{n}_{11}$

\subsection{Multicast Admission Control}

Most of the existing schemes depend on individual receivers to detect congestion and adjust their receiving rates which are much disadvantageous. We propose a scheme which adjusts the multicast traffic rate at each bottleneck of a multicast tree. Each node estimates its current traffic load and arrival rate. Based on its traffic load, it estimates the receiving rate. If the receiving rate is less than the arrival rate, it adaptively adjusts its receiving rate.

In order to adjust the total number of multicast flows which traverse a bottleneck, the following procedure is used. In our proposed scheme, based on the link's output queue state, multicast flows at a bottleneck can be blocked or released. Let the number of packets in the queue is N. Let PT1 and PT2 (PT1 < PT2) are two thresholds for the queue size. Then the flow is released or blocked based on the following conditions.

If $\mathrm{N}<=\mathrm{PT} 1$, then the multicast flow is released.

If $\mathrm{N}>\mathrm{PT} 2$, then the multicast flow is blocked.

In most of the existing schemes, in order to detect congestion and for adjusting the receiving rate they depend on the individual receivers. In our proposed scheme multicast traffic rate is adjusted at each bottleneck of a multicast tree. Whenever congestion happens or about to, then the multicast sessions which traverse the branch are blocked. Thus the packets are stopped from entering the branch. The blocked flows are released to traverse the branch when the branch is lightly utilized.

\subsection{Multicast Traffic Rate Adjustment}

When the available bandwidth is less than the required bandwidth or the queue size is less than a minimum threshold value, it indicates the possibility of congestion or packet loss. The behaviour of the multicast session is expressed as

$$
\begin{array}{rlrl}
R(t+1)= & \{R(t)-g & R(t)>B \\
& R(t)+g & & R(t)<=B \\
& R(t) & & \text { otherwise }\}
\end{array}
$$

Here $\mathrm{R}(\mathrm{t})$ denotes the instantaneous rate of the multicast session at time $\mathrm{t}$.

$\mathrm{B}$ is the bottleneck bandwidth.

When $\mathrm{R}(\mathrm{t})>\mathrm{B}$ then the network is congested and the multicast session decreases its rate by a step $\mathrm{g}$.

If $\mathrm{R}(\mathrm{t})<=\mathrm{B}$ then the network is not congested and the multicast session increases its rate by a step $\mathrm{g}$.

The proposed scheme overcomes most of the disadvantages of existing schemes:

1. Link errors cannot cause the proposed scheme to wrongly block a layer, because instead of the loss information at receivers, the queue state at a bottleneck is used as the metric to adjust the multicast traffic rate at the bottleneck.

2. Link access delay caused by competition in MANETs cannot hinder the rate adjustment in this scheme, because, it blocks multicast layers right at each bottleneck of a multicast tree instead of depending on receivers to request pruning to drop layers.

3. Because of the on-the-spot information collection and rate control this scheme has very limited control traffic overhead.

Moreover, the proposed scheme does not impose any significant changes on the queuing, scheduling or forwarding policies of existing networks. 


\section{Simulation Results}

\section{Simulation Model and Parameters}

We use NS2 to simulate our proposed protocol. In our simulation, the channel capacity of mobile hosts is set to the same value: 2 Mbps. We use the distributed coordination function (DCF) of IEEE 802.11 for wireless LANs as the MAC layer protocol. It has the functionality to notify the network layer about link breakage.

In our simulation, 50 mobile nodes move in a 1000 meter x 1000 meter region for 50 seconds simulation time. We assume each node moves independently with the same average speed. All nodes have the same transmission range of 250 meters. In our simulation, the minimal speed is $5 \mathrm{~m} / \mathrm{s}$ and maximal speed is $5 \mathrm{~m} / \mathrm{s}$. The simulated traffic is Constant Bit Rate (CBR). Our simulation scenario table1:

\begin{tabular}{|l|l|}
\hline No. of Nodes & 50 \\
\hline Area Size & $1000 \mathrm{X} 1000$ \\
\hline Mac & 802.11 \\
\hline Radio Range & $250 \mathrm{~m}$ \\
\hline Simulation Time & $50 \mathrm{sec}$ \\
\hline Traffic Source & CBR \\
\hline Packet Size & $250,500, \ldots 1000$ \\
\hline Mobility Model & Random Way point \\
\hline Speed & $5 \mathrm{~m} / \mathrm{s}$ \\
\hline Receivers & $5,10, \ldots 25$ \\
\hline Pause time & $5 \mathrm{~s}$ \\
\hline Transmit Power & $0.660 \mathrm{w}$ \\
\hline Receiving Power & $0.395 \mathrm{w}$ \\
\hline Idle Power & $0.335 \mathrm{w}$ \\
\hline Initial energy & $3.1 \mathrm{~J}$ \\
\hline
\end{tabular}
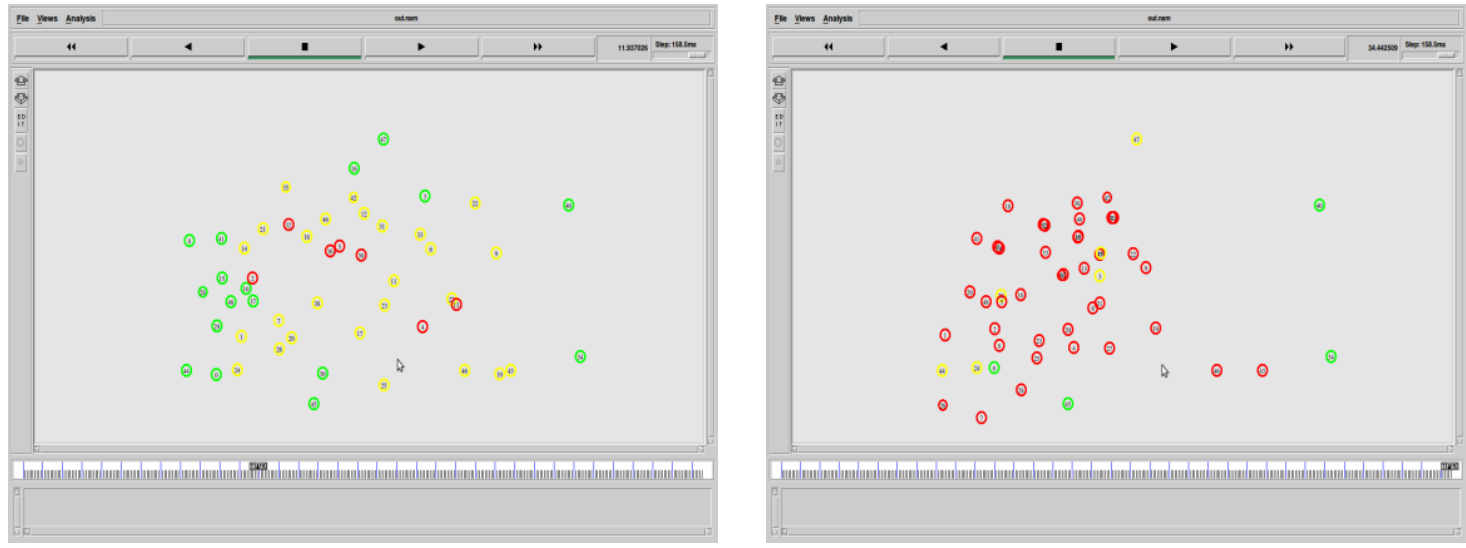

Fig 4.1 Snapshots of NAM identifying the energy nodes

\section{Performance Metrics}

We compare our EEMCC protocol with the multicast AODV [14] protocol. We evaluate mainly the performance according to the following metrics.

Average end-to-end delay: The end-to-end-delay is averaged over all surviving data packets from the sources to the destinations.

Average Packet Delivery Ratio: It is the ratio of the No. of packets received successfully and the total no. of packets sent.

Average Energy Consumption: The average energy consumed by the nodes in receiving and sending the packets are measured.

Throughput: It is the number of packets received by all the nodes in the network. 


\section{Results \\ Analysis of QOS parameters based on number of Receivers}

In this analysis, the number of receivers per group considered as $5,10 \ldots . .25$. When the numbers of receivers are increased, the variation of delay for both AODV and EEMCCP. The delay required for packet transmission is less for EEMCCP, when compared to AODV. As seen in the graph the delivery ratio is high for EEMCCP, when compared to AODV. energy consumption for both the cases. As seen from the figure, the energy consumption is less for EEMCCP, when compared to AODV.

The graphs show the throughput occurred for both the cases. The throughput is high for EEMCCP, when compared to AODV. The graph shows the overhead packets occurred for both the cases. From this the overhead is low for EEMCC, when compared to AODV.

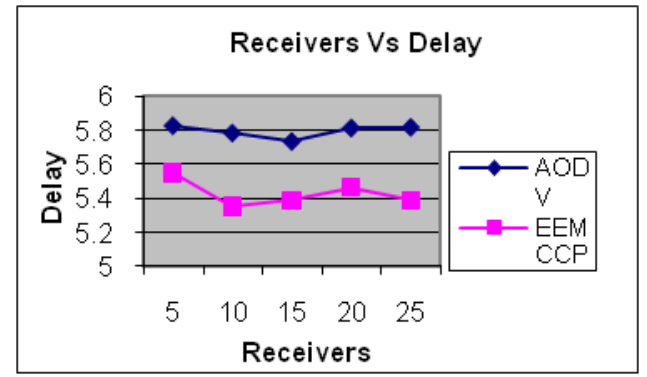

Fig 4.2 Receivers Vs Delay

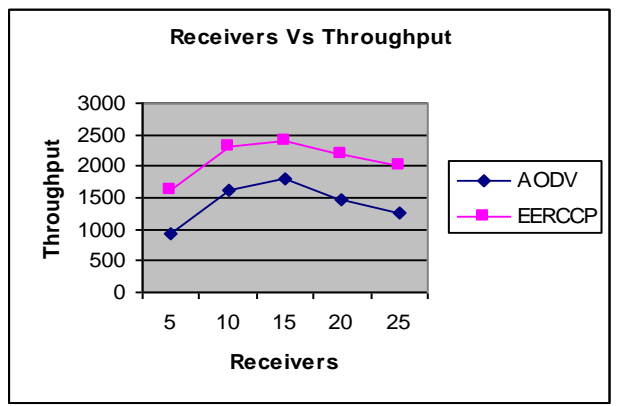

Fig 4.4Receivers Vs Throughput

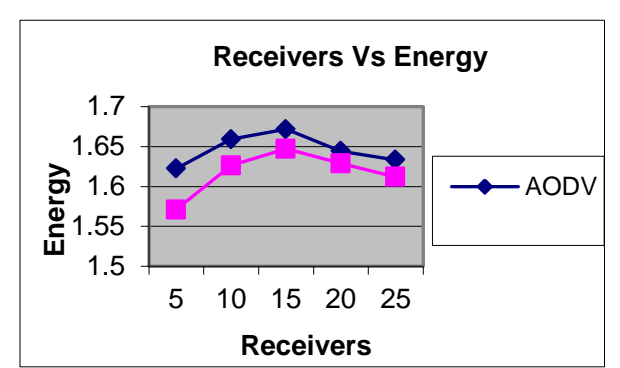

Fig 4.3 Receivers Vs Energy

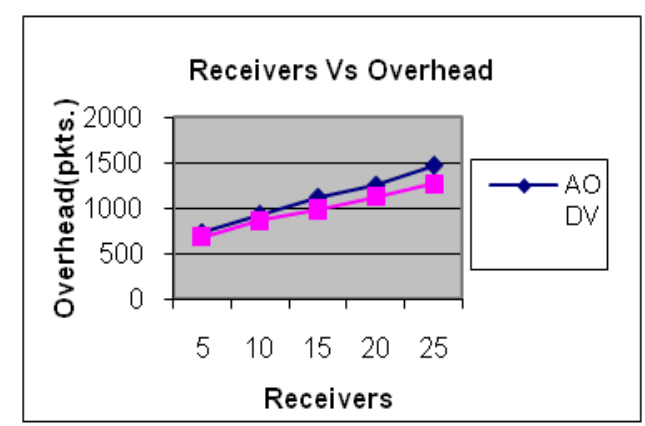

Fig 4.5 Receivers Vs Overhead

\section{Analysis of QoS parameters based on Packet size}

In this experiment, the packet size considered as $250,500 \ldots . .1000$ bits., it is observed that there is a reduction in delay, traffic overhead and energy consumption, improvement in delivery ratio and throughput in EEMCC when compared to AODV.

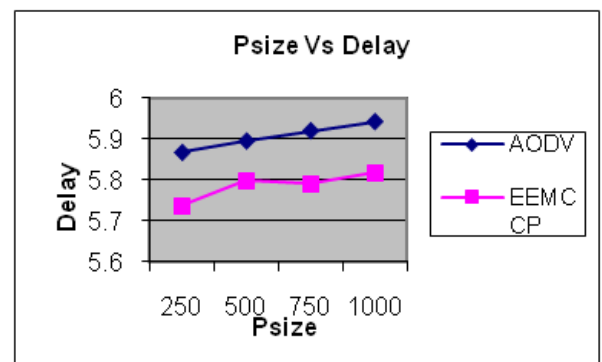

Fig 4.6 Packet size vs Delay

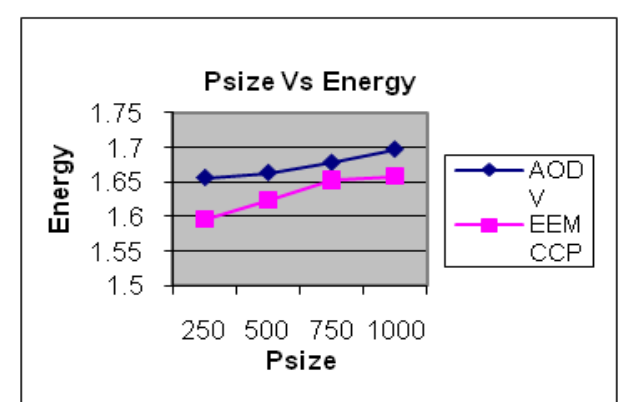

Fig 4.7 Packet size vs Energy 


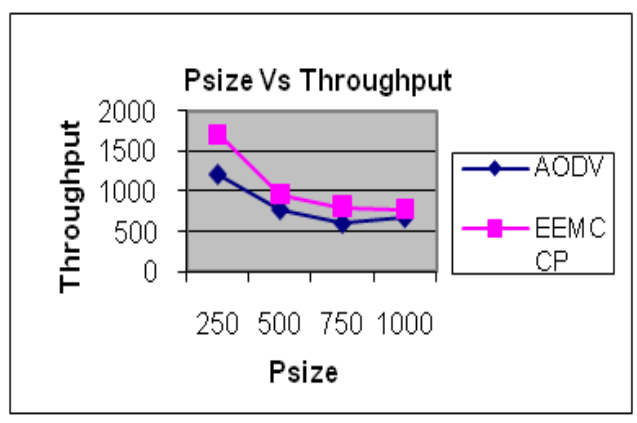

Fig 4.8 Packet size vs Throughput

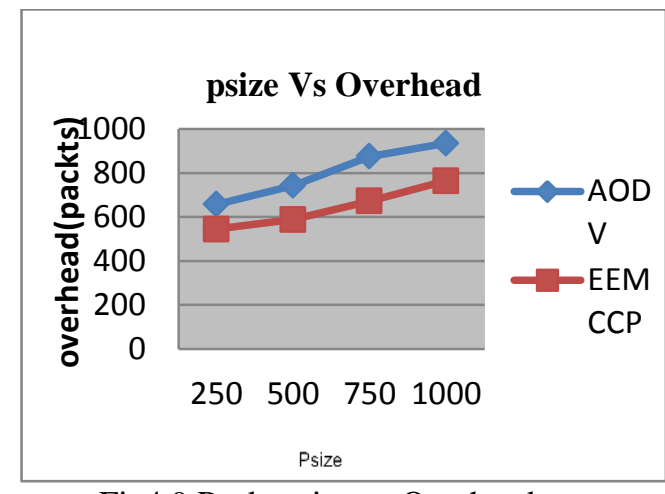

Fig4.9 Packet size vs Overhead

\section{v. Conclusion}

In this paper, we have proposed an energy efficient and reliable congestion control protocol for multicasting in mobile adhoc networks. Our proposed protocol overcomes the disadvantages of existing multicast congestion control protocols which depend on individual receivers to detect congestion and adjust their receiving rates. Because of the on-the-spot information collection and rate control, this scheme has very limited control traffic overhead and delay. Moreover, the proposed scheme does not impose any significant changes on the queuing, scheduling or forwarding policies of existing networks. Simulation results have shown that our proposed protocol has better delivery ratio and throughput with less delay and energy consumption when compared with existing protocol.

\section{References}

[1]. LuoJunhai, Xue Liu, Ye Danxia," Research on multicast routing protocols for mobilead-hoc networks", Elsevier, 2007

[2]. AbdussalamNuriBaryun, and Khalid Al-Begain," A Design Approach for MANET Multicast Protocols", ISBN, 2008

[3]. Hua Chen, Baolin Sun," An Entropy-Based Fuzzy Controllers QoS Routing Algorithm in MANET",IEEE,2009

[4]. TolgaNumanoglu and Wendi Heinzelman," Improving QoS in Multicasting Through

[5]. Adaptive Redundancy",University of Rochester Center for Electronic Imaging Systems

[6]. D. Agrawal, T. Bheemarjuna Reddy, and C. Siva Ram Murthy," Robust Demand-Driven Video Multicast over Ad hoc Wireless Networks",IEEE,2006

[7]. Guojun Wang, Jiannong Cao, Lifan Zhang, Keith C. C. Chan," A Novel QoS Multicast Model in Mobile Ad Hoc Networks", IEEE International Parallel and Distributed Processing Symposium,2005

[8]. Vida Lashkari B. O., Mehdi Dehghan" QoS-aware Multicast Ad hoc On-Demand

[9]. Distance Vector Routing”, WCE 2007

[10]. Zeyad M. Alfawaer, GuiWei Hua, and Noraziah Ahmed," A Novel Multicast Routing Protocol for Mobile Ad Hoc Networks", ISSN, 2007

[11]. HaraldTebbe and Andreas J. Kassler ," QAMNet: Providing Quality of Service to Ad-hoc Multicast Enabled Networks",Wireless Pervasive Computing, 2006 1st International Symposium on, IEEE,2006

[12]. Mohammed Saghir, Tat-Chee Wan, Rahmat Budiarto," QoS Multicast Routing Based on Bandwidth Estimation in Mobile Ad Hoc Networks", ICCCE2006

[13]. RavindraVaishampayan, J.J. Garcia-Luna-Aceves,” Efficient and Robust Multicast Routing in Mobile Ad Hoc Networks", IEEE, 2004

[14]. Y. Chen and Y. Ko, “A Lantern-Tree Based QoS on Demand Multicast Protocol for A wireless Ad hoc Networks," IEICE Trans. Communications, vol. E87-B, 2004

[15]. 13 N. Ben Ali, A. Belghith, J. Moulierac, M. Molnar," QoS multicast aggregation under multiple additive constraints”, Elsevier, 2008

[16]. Elizabeth M. Royer, Charles E. Perkins," Multicast Operation of the Ad-hoc On-Demand Distance Vector Routing Protocol",ACM,1999 